D.T. Wickramasinghe, L. Ferrario, and G.V. Bicknell, eds.

\title{
Clues and Modelling for Missing Boundary Layer in Cataclysmic Variables
}

\author{
M. Huang \\ Villanova University, Villanova, PA 19085, USA
}

W.M. Sparks

Los Alamos National Lab, Los Alamos, NM 87545, USA

\author{
E.M. Sion
}

Villanova University, Villanova, PA 19085, USA

\begin{abstract}
.
Recently, it has become observationally evident that during a dwarf nova (DN) outburst, a significant portion of the gravitational energy accreted onto the white dwarf (WD) may not be radiated away instantly from a narrow boundary layer as predicted by the standard disk theory. Instead, it may be stored in the WD through various mechanisms and the radiative area may be much larger; thus the long-puzzling missing boundary layer may be accounted for when the response of the WD to the accretion is considered. The results from our group and collaborators on this aspect are outlined in the first part. A progress report on the development of a new numerical model forms the second.
\end{abstract}

\section{Observational Clues}

The standard $\alpha$-model theory (Pringle 1981) predicts that, during a DN outburst, the boundary layer between the WD surface and the inner disk radiates away $\sim \frac{1}{2}$ of the accreted energy from an area $\sim 10^{-4} A_{W D}\left(A_{W D}=\right.$ the area of WD stellar disc) and has an effective temperature as high as $500,000 \mathrm{~K}$, which is not observed. This discrepancy is a long-standing problem in astrophysics and is often referred as Missing Boundary Layer problem.

We examined two basic assumptions made in the standard theory:

Assumption I. The gravitational energy accreted is released instantly. Sion (1995) investigated models with realistic WD physics and pointed out that through compressional heating, a significant portion $(\sim 20-30 \%)$ of the energy accreted during an outburst is stored in the interior of the WD, some of which may never be released and heats the WD secularly. Another mechanism of storing the accreted energy, shear mixing, is investigated by Sparks et al. (1993), who point out that the forced convection under the strong shearing in the boundary layer during an outburst carries the energy deep down the WD atmosphere. Models including this effect give a longer cooling time scale of the WD during the following quiescence, as observed. 
Assumption II. The rotational speed at the inner edge of the disk matches that on the WD surface. The boundary condition used in the terrestrial hydrodynamic problem has the same name: a slipless rigid wall. One consequence of this boundary condition is that the area of the boundary layer is very small: $\frac{A_{W P}}{\mathcal{M}^{2}}$ ( $\mathcal{M}=\frac{V_{K}}{c_{s}} \sim 10^{2}$ is the Mach number of the Keplerian speed on the WD surface). It may be misleading to apply this boundary condition to DNe, in which the surface of the WD is gaseous. Experiments of two shearing gas streams show that the shearing zone is much wider (Gutmark, Schadow \& Yu 1995).

Analysis of HST (Huang et al. 1996, and Sion et al. 1996) and IUE (Gänsicke \& Beuermann 1996) spectra of VW Hyi during quiescences show strong evidences of a hot belt on the WD surface with area about $\sim 0.1 A_{W D}$ and rotational speed close to $V_{K}$. We conclude that this is a remnant of the strong shearing during the previous outburst. This almost directly shows that during an outburst, the equatorial zone of the WD surface joining the inner disk is actually spun up to match the disk speed!

\section{A Two Dimensional Model (Progress Report)}

The previous simulations by Sparks et al. (1993) and Sion (1995) are one dimensional (1-D). The recent identification of the 2-D belt structure on the WD surface in VW Hyi mentioned above urgently demands more realistic simulations using a 2-D model, which is under development in our group. To allow for model time steps exceeding the limit set by the Courant condition, the sound-crossing time in a grid cell inside the degenerate WD, the code has to be implicit for numerical stability.

Using an energy criteria of shear mixing characterized by Richardson num$b e r$, we find that the forced convection is most efficient along the equi-potential surface of the differentially rotating WD atmosphere. To simulate this effect, we developed a new time-dependent zoning scheme in which the time-dependent grid follows the equi-potential surfaces. Further results will be reported in due time.

\section{References}

Gänsicke, B.T. and Beuermann, K. 1996, A\&A, 309, L47

Gutmark, E.J., Schadov, K.C. \& Yu, K.H., Ann. Rev. of Fluid Mechanics, 1995, 27,375

Huang, M., Sion, E.M., Hubeny, I., Cheng, F.H. \& Szkody, P. 1996, AJ, 111, 2386

Pringle, J.E. 1981, Ann. Rev. Ast. Astrophy., 19, 137

Sion, E.M. 1995, ApJ, 438, 876

Sion, E.M., Cheng, F.H., Huang, M., Hubeny, I., \& Szkody, P. 1996, ApJ, in press

Sparks, W.M., Sion, E.M., Starrfield, S.G., and Austin, S., 1993, Cataclysmic Variables and related Physics (Edited by O. Regev and G. Shaviv), p96. 